\title{
Recent results from the strong interaction programme of the NA61/SHINE experiment
}

\section{Grzegorz Stefanek* for the NA61/SHINE Collaboration}

Jan Kochanowski University in Kielce, Poland

E-mail: grzegorz.stefanek@ujk.edu.pl

The exploration of the QCD phase diagram $\left(\mathrm{T}-\mu_{B}\right)$ particularly the search for a phase transition from hadronic to partonic degrees of freedom and the critical point is one of the most challenging theoretical and experimental tasks in present heavy ion physics. Unfortunately the QCD predictions are to a large extent qualitative, as QCD phenomenology at finite temperature and baryon number is one of the least explored domains of the theory.

The NA49 study of central collisions of heavy nuclei at CERN SPS energies revealed rapid changes in the energy dependence of hadron production properties in the narrow range arround $30 \mathrm{~A} \mathrm{GeV}$. The results indicate the onset of deconfinement in the early stage of the produced system and motivate similar studies by the NA61/SHINE experiment and the ongoing search for the predicted critical point. NA61/SHINE results on particle production properties as well as eventby-event fluctuations in $\mathrm{p}+\mathrm{p}, \mathrm{Be}+\mathrm{Be}$ and $\mathrm{Ar}+\mathrm{Sc}$ collisions are shown as a function of beam energy and system size. The observation of rapid change of hadron production properties that starts when moving from light to intermediate mass ion collisions might be interpreted as the beginning of the formation of large clusters of strongly interacting matter - the onset of fireball. The future ion program of the NA61/SHINE experiment including planned measurements of charm hadrons, mostly $\mathrm{D}$ mesons, production in $\mathrm{Pb}+\mathrm{Pb}$ collisions is presented.

The 39th International Conference on High Energy Physics (ICHEP2018)

4-11 July, 2018

Seoul, Korea

${ }^{*}$ Speaker. 


\section{Introduction}

To access a broad region of the QCD phase diagram one can vary the temperature and baryon chemical potential of the nuclear matter created in the collisions by tuning the beam energy and size of colliding nuclei. Such a comprehesive two dimensional scan of the phase diagram was started by the NA61/SHINE experiment in 2009 by collecting $p+p$ interactions at six SPS energies and it will be completed in 2018 by data taking of $\mathrm{Pb}+\mathrm{Pb}$ collisions. It mainly focuses on the study of hadron spectra, the properties of the onset of deconfinement and the search for the critical point.

\section{Onset of deconfinement and onset of fireball}

The first energy scan at SPS was conducted by NA49 the predecessor of the NA61/SHINE experiment [1]. It collected central $\mathrm{Pb}+\mathrm{Pb}$ collisions at $\mathrm{SPS}$ beam energies from 20 to $158 \mathrm{GeV}$ per nucleon. These data show that the $\left\langle\mathrm{K}^{+}\right\rangle /\left\langle\pi^{+}\right\rangle$ratio exhibits a step rise from the threshold of kaon production, a maximum around the low SPS energy and a decrease to lower plateau value at higher energy [2]. Such energy dependence, which is called horn, agrees with the predictions of the Statistical Model of the Early Stage [3] in which it is related to the decrease of the mass of strangeness carriers and the ratio of strange to non-strange degrees of freedom. NA61/SHINE data show no horn structure in $\mathrm{p}+\mathrm{p}$ and $\mathrm{Be}+\mathrm{Be}$ collisions at SPS energies. They agree with $\mathrm{p}+\mathrm{p}$ world data and suggest both systems behave quite similar (Fig. 1). The values for $\mathrm{Ar}+\mathrm{Sc}$ collisions lay between light and heavy systems (Fig. 1 (middle)).

Other structure called step is observed in the energy dependence of the inverse slope parameter (T) for $m_{T}$ distribution of $K^{+}, K^{-}$mesons in central $\mathrm{Pb}+\mathrm{Pb}$ collisions (Fig. 1 (right)). The dependence, which resembles the structure observed on phase diagram of water, is interpreted as the effect of softening Equation of State at mixed-phase which weakens transverse expansion. The existence of step structure in $\mathrm{p}+\mathrm{p}, \mathrm{Be}+\mathrm{Be}$ collisions measured by NA61/SHINE is still under investigation.
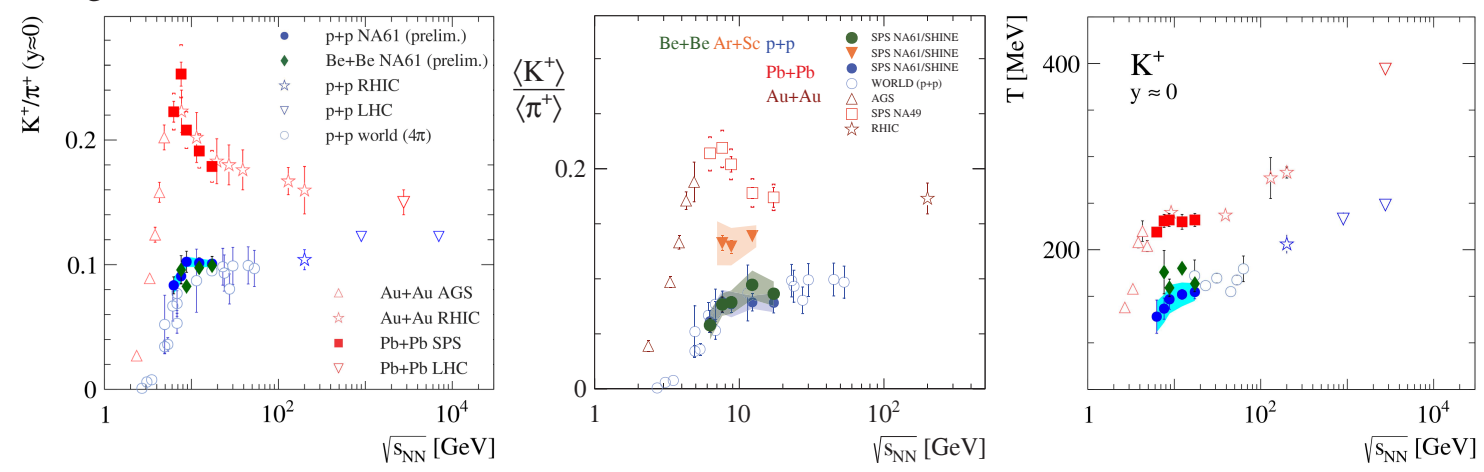

Figure 1: The energy dependence of the $\left\langle\mathrm{K}^{+}\right\rangle /\left\langle\pi^{+}\right\rangle$yeald ratio at midrapidity (left) and in the full phase space (middle) - horn, as well as the inverse slope parameter of the trasverse mass distribution for $\mathrm{K}^{+}$at midrapidity (right) - step

The well known prediction of the Statistical Model with Ideal Boltzmann Gas within Canonical and Grand Canonical Ensembles is the canonical suppresion of the mean multiplicity as a function of the mean multiplicity within Grand Canonical Ensemble which is proportional to the cluster volume [4]. This qualitative expectation confronted with NA49, NA61/SHINE data which 
show the rapid change of the $\left\langle\mathrm{K}^{+}\right\rangle /\left\langle\pi^{+}\right\rangle$ratio at midrapidity (Fig. 2 (left)) suggests there are two regimes: Wounded Nucleon Model regime for light systems with incoherent superposition of particles from wounded nucleons and Statistical Model regime for heavier systems with large clusters. The jump between light and intermediate or heavy systems can be interpreted as the onset of fireball.
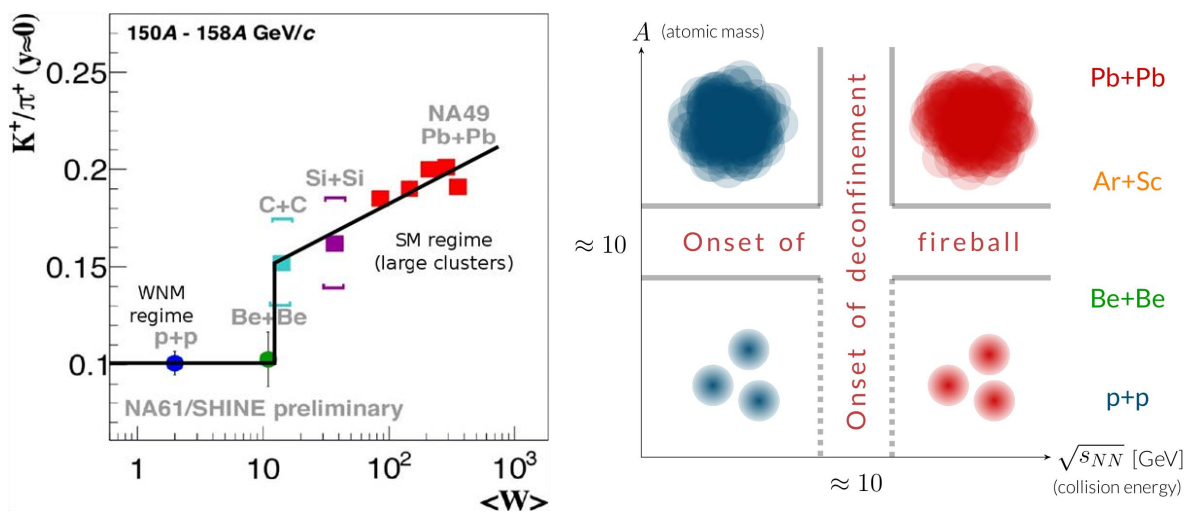

Figure 2: Left: System size dependence of the $\left\langle\mathrm{K}^{+}\right\rangle /\left\langle\pi^{+}\right\rangle$ratio at midrapidity for $\mathrm{p}+\mathrm{p}, \mathrm{Be}+\mathrm{Be}, \mathrm{C}+\mathrm{C}, \mathrm{Si}+\mathrm{Si}$ and $\mathrm{Pb}+\mathrm{Pb}$ collisions at $150 \mathrm{~A}, 158 \mathrm{~A} \mathrm{GeV} / \mathrm{c}$. Right: Two-dimentional sketch of four domains of hadron production separated by two thresholds: the onset of deconfinement and the onset of firebal, as indicated by the NA61/SHINE scan.

Scans conducted by NA49, NA61/SHINE together indicate four domains of hadron production separated by two thresholds: the onset of deconfinement and the onset of fireball (Fig. 2 (right)). The onset of deconfinement is well established in central $\mathrm{Pb}+\mathrm{Pb}(\mathrm{Au}+\mathrm{Au})$ collisions. Its presence in collisions of light nuclei, in particular inelastic $p+p$ interations is questionable. The onset of fireball is observed moving from light to intermediate and heavy systems.

\section{Critical point and critical fluctuations}

The theory predics characterictic features of a second order phase transition at the critical point or line. One of them is the divergence of the correlation length which means that the system becomes scale invariant. This leads to large fluctuations of particle multiplicity and other charcteristics of events. One of the goals of the NA61/SHINE scanning program is to find an evidence for the critical point as a maximum of the event-by-event fluctuations in analogy to the critical opalescence in the conventional matter.

In the experimental search for the critical point one should use proper measures of fluctuations especially strongly intensive measures which are independent on the volume and its fluctuations. Such measures are not sensitive to material conservation laws. Two exemplary strongly intensive measures are $\Delta\left[P_{T}, N\right], \Sigma\left[P_{T}, N\right]$ in which $P_{T}$ and $N$ are the sum of the absolute values of particle trasverse momenta $p_{T}$ and particle multiplicity respectively. No indication for the critical point is observed so far (Fig. 3 (left)) in this NA61/SHINE analysis.

However, the NA49 intermittency analysis of the second factorial moment of the number of pion pairs and protons in $p_{T}$ space indicate the presence of power law fluctuations approaching in size the prediction of critical QCD [5, 6]. Similar analysis of the NA61/SHINE data is ongoing. 


\section{Open charm measurements}

The NA61/SHINE charm program is a natural extension of the previous studies to the new domain of $c$-quark mass to study the mechanism of charm production, the influence of the onset of deconfinement on charm production and the impact of the formation of quark-gluon plasma on $J / \psi$ production. NA61/SHINE aims to measure the production of $c \bar{c}$ pairs in the full phase space in the coming years and expects to narrow the spectrum of viable theoretical models.
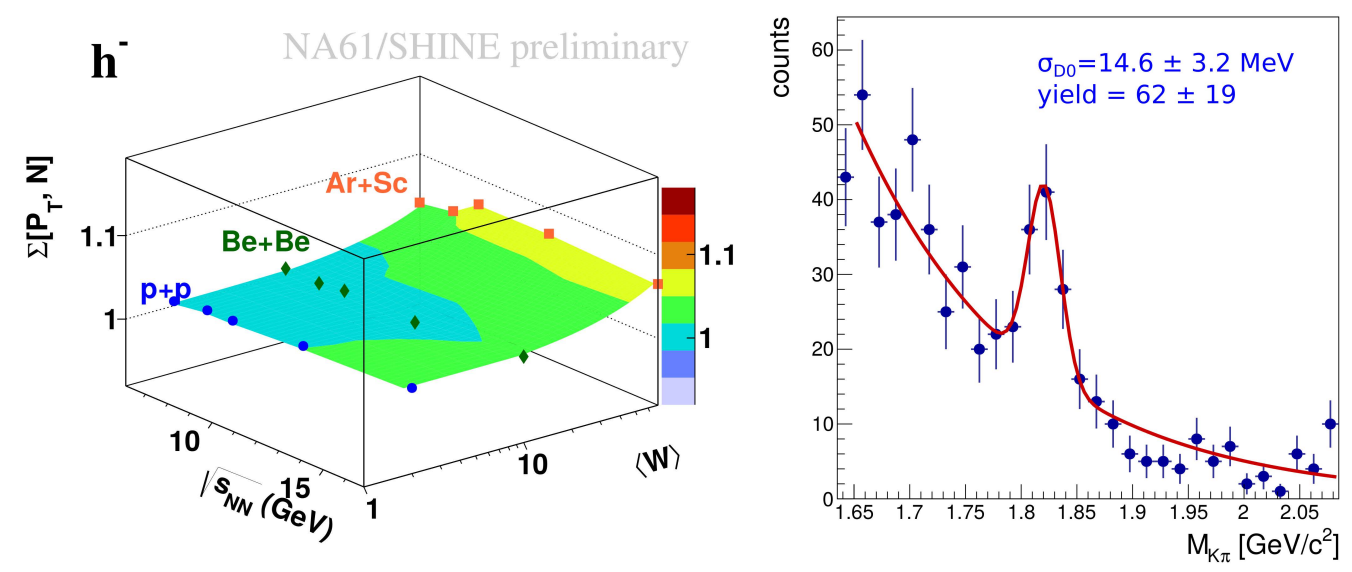

Figure 3: Left: $\Sigma\left[P_{T}, N\right]$ measured by NA61/SHINE in inelastic $\mathrm{p}+\mathrm{p}$ interactions and violent $\mathrm{Be}+\mathrm{Be}, \mathrm{Ar}+\mathrm{Sc}$ collisions at the CERN SPS energies. Results refer to negatively charged hadrons at forward rapidity $(0<$ $y_{\pi}<y_{\text {beam }}$ ) and $p_{T}<1.5 \mathrm{GeV} / \mathrm{c}$. Right: Invariant mass distribution of unlike charge $\pi, \mathrm{K}$ decay track candidates. The spectrum was obtained by applying background reduction cuts to enhance $D^{0}$ signal.

The measurements of open charm production started in december 2016 using newly constructed Small Acceptance Vertex Detector [7]. The detector measures the decay vertices of $D^{0}$ mesons. The first observation of $D^{0}$ peak in pilot data on $\mathrm{Pb}+\mathrm{Pb}$ collisions at top SPS energy (Fig. 3 (right)) demonstrates that the detector can be used for precise $D^{0}$ production measurements.

\section{Acknowledgements}

This work was supported by the Polish National Science Centre grants 2015/18/M/ST2/00125, 2016/23/B/ST2/00692.

\section{References}

[1] N. Abgrall et al. [NA61/SHINE Collaboration], JINST 9, P06005 (2014).

[2] C. Alt et al. [NA49 Collaboration], Phys. Rev. C 77, 024903 (2008).

[3] M. Gazdzicki and M. I. Gorenstein, Acta Phys. Polon. B 30, 2705 (1999).

[4] J. Rafelski and M. Danos, Phys. Lett. 97B, 279 (1980).

[5] T. Anticic et al. [NA49 Collaboration], Phys. Rev. C81, 064907 (2010).

[6] T. Anticic et al. [NA49 Collaboration], Eur. Phys. J. C 75, no. 12, 587 (2015).

[7] M. Deveaux et al. [NA61/SHINE Collaboration], EPJ Web Conf. 171, 10003 (2018). 